CRYSTALLOGRAPHIC COMMUNICATIONS

ISSN 2056-9890

Received 27 June 2016

Accepted 9 August 2016

Edited by H. Ishida, Okayama University, Japan

Keywords: crystal structure; pentamethylcyclopentadienyl; olefin complex; cobalt.

CCDC reference: 1498272

Supporting information: this article has supporting information at journals.iucr.org/e

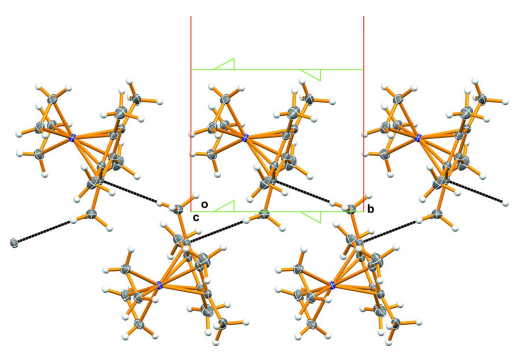

OPEN $\odot$ ACCESS

\section{Crystal structure of bis $\left(\eta^{2}\right.$-ethylene $)\left(\eta^{5}\right.$-penta- methylcyclopentadienyl)cobalt}

\author{
Chandika D. Ramful, Katherine N. Robertson and Kai E. O. Ylijoki* \\ Department of Chemistry, Saint Mary's University, 923 Robie St., Halifax, NS, B3H 3C3, Canada. *Correspondence \\ e-mail: kai.ylijoki@smu.ca
}

The title compound, $\left[\mathrm{Co}\left(\mathrm{C}_{10} \mathrm{H}_{15}\right)\left(\mathrm{C}_{2} \mathrm{H}_{4}\right)_{2}\right]$, was prepared by Na/Hg reduction of $\left[\mathrm{Co}_{2}\left(\mathrm{C}_{10} \mathrm{H}_{15}\right)_{2}(\mu-\mathrm{Cl})_{2}\right]$ in THF under an ethylene atmosphere and crystallized from pentane at $193 \mathrm{~K}$. The $\mathrm{Co}-\mathrm{C}$ (olefin) bonds have an average length of 2.022 (2) $\AA$, while the $\mathrm{Co}-\mathrm{C}$ (pentadienyl) bonds average 2.103 (19) $\AA$. The olefin $\mathrm{C}=\mathrm{C}$ bonds are 1.410 (1) $\AA$. The dihedral angle between the planes defined by the cyclopentadienyl ligand and the two olefin ligands is $0.25(12)^{\circ}$. In the crystal, molecules are linked into chains by $\mathrm{C}-\mathrm{H} \cdots \pi$ interactions.

\section{Chemical context}

The title compound, $\mathrm{Cp} * \mathrm{Co}\left(\mathrm{CH}_{2} \mathrm{CH}_{2}\right)_{2}\left(\mathrm{Cp}^{*}=\right.$ pentamethylcyclopentadienyl), was first reported in 1981 by Spencer and coworkers (Beevor et al., 1981) in their quest to find a more thermally labile analogue of the related $\mathrm{Cp} * \mathrm{Co}$ (dicarbonyl) complex. Since this first report, it and other olefin complexes of cobalt with $\mathrm{Cp}^{*}$ or $\mathrm{Cp}(\mathrm{Cp}=$ cyclopentadienyl) have become important precursors for the generation of $\mathrm{Cp}^{\prime} \mathrm{Co} L(L$ $=$ olefin, pyridine, etc $)$ and $\mathrm{Cp}^{\prime} \mathrm{Co}$ fragments used as active species in $\mathrm{C}-\mathrm{H}$ bond activation (Lenges et al., 1997, 1998, 2000; Broere \& Ruijter, 2012), cyclotrimerization of alkynes (Dosa et al., 2002; Holmes et al., 2015) and C-S bond activation (Jones \& Chin, 1994; Chan et al., 2015). The utility of the $\mathrm{Cp} * \mathrm{Co}\left(\mathrm{CH}_{2} \mathrm{CH}_{2}\right)_{2}$ complex in organometallic synthesis has been explored extensively. Examples include the preparation of high-oxidation state $\mathrm{Co}^{\mathrm{V}}$ complexes (Brookhart et al., 2000) and the preparation of $\mathrm{Cp} * \mathrm{Co}\left(\eta^{5}\right.$-pentadienyl) ${ }^{+}$complexes (Witherell et al., 2008; Ylijoki et al., 2009, 2015).

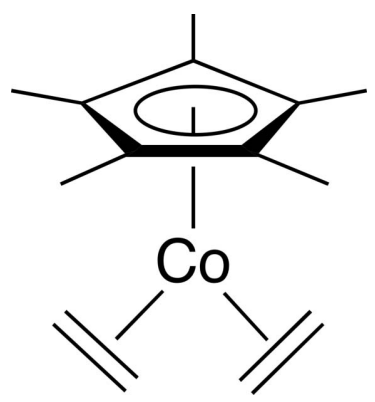

\section{Structural commentary}

The molecular structure of the title compound is shown in Fig. 1. Although the cobalt atom is located on a general position, the molecule is essentially $C_{2 \mathrm{v}}$ symmetric, which agrees with the symmetry of the ${ }^{1} \mathrm{H}$ NMR data (Beevor et al., 


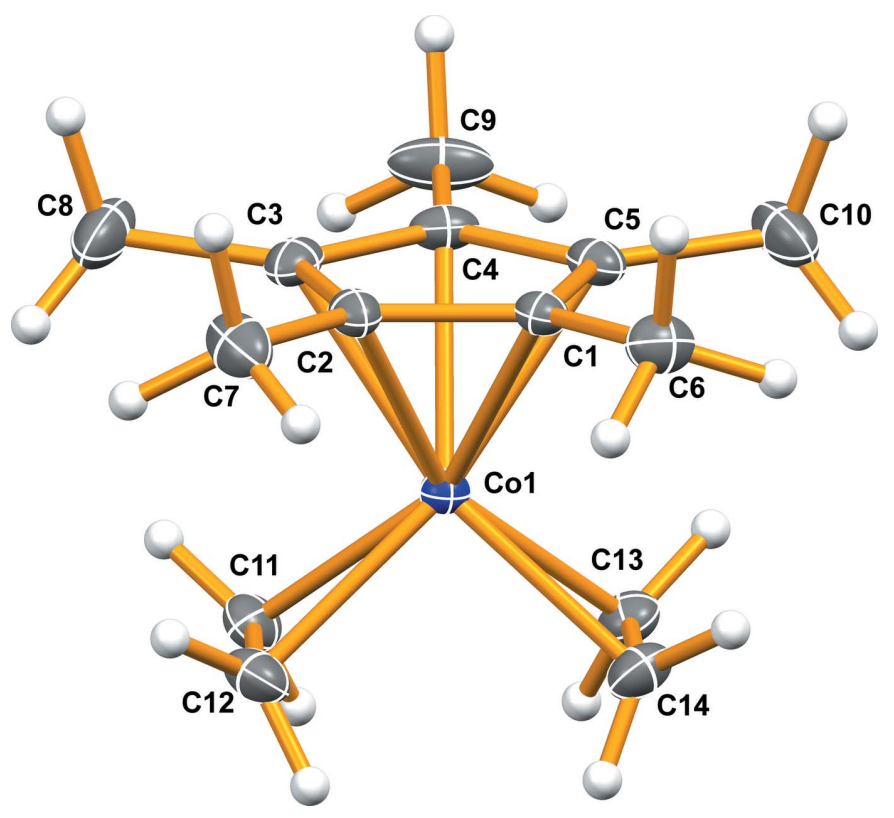

Figure 1

The molecular structure of the title compound, showing the atom labelling. Displacement ellipsoids are drawn at the $50 \%$ probability level for non-H atoms.

1981; Nicholls \& Spencer, 1990). The Co-C(olefin) bonds have an average length of $2.022(2) \AA$, while the $\mathrm{Co}-\mathrm{C}\left(\mathrm{Cp}^{*}\right)$ bonds average 2.103 (19) $\AA$. The olefin $\mathrm{C}=\mathrm{C}$ bonds are 1.410 (1) $\AA$. All bond lengths are in agreement with those reported for the related $\mathrm{Cp} *$ Cobis(trimethylvinylsilane) complex (Lenges et al., 1998). The C11-Co1-C14 and C12Co1-C13 bond angles average $104.64(1)^{\circ}$, indicating a parallel arrangement of the olefin ligands. The dihedral angle between the planes defined by the $\mathrm{Cp}^{*}$ ligand ( $\left.\mathrm{C} 1-\mathrm{C} 5\right)$ and the two olefin ligands $(\mathrm{C} 11-\mathrm{C} 14)$ is $0.25(12)^{\circ}$.

\section{Supramolecular features}

In the crystal, a weak $\mathrm{C}-\mathrm{H} \cdots \pi$ interaction is observed between one of the methyl groups and the $\mathrm{Cp}^{*}$ ring edge of the adjacent molecule related by a $2_{1}$ screw axis. The shortest contact occurs between the $\mathrm{C} 6-\mathrm{H} 6 \mathrm{C}$ of the methyl group and the $\mathrm{C} 1$ atom of the $\mathrm{Cp}^{*}$ ring $\left[\mathrm{H} 6 \mathrm{C} \cdots \mathrm{C} 1^{\mathrm{i}} 2.79, \mathrm{C} 6 \cdots \mathrm{Cl}^{\mathrm{i}}\right.$ 3.734 (3) $\AA$, C6-H6C $\cdots \mathrm{C}^{\mathrm{i}} 162^{\circ}$; symmetry code (i): $-x, y-\frac{1}{2}$, $-z+\frac{1}{2}$ ], while the $\mathrm{H} 6 C \cdots \mathrm{Cp} *$ ring centroid distance is $3.00 \AA$. The molecules are linked through the $\mathrm{C}-\mathrm{H} \cdots \pi$ interactions, forming a helical chain parallel to the $b$ axis (Fig. 2).

\section{Database survey}

The Cambridge Structural Database (CSD, Version 5.37; Groom et al., 2016) contains one additional example of a Cp*Cobis(olefin) complex: the Cp*Cobis(trimethylvinylsilane) complex (GIQHUJ) reported by Brookhart and coworkers (Lenges et al., 1998). The title compound is isostructural with the $\mathrm{Cp} * \mathrm{Fe}\left(\mathrm{CH}_{2} \mathrm{CH}_{2}\right)_{2}$ complex (VOGJAD; Fig. 3) reported by Fürstner et al. (2008). The iron compound crys-

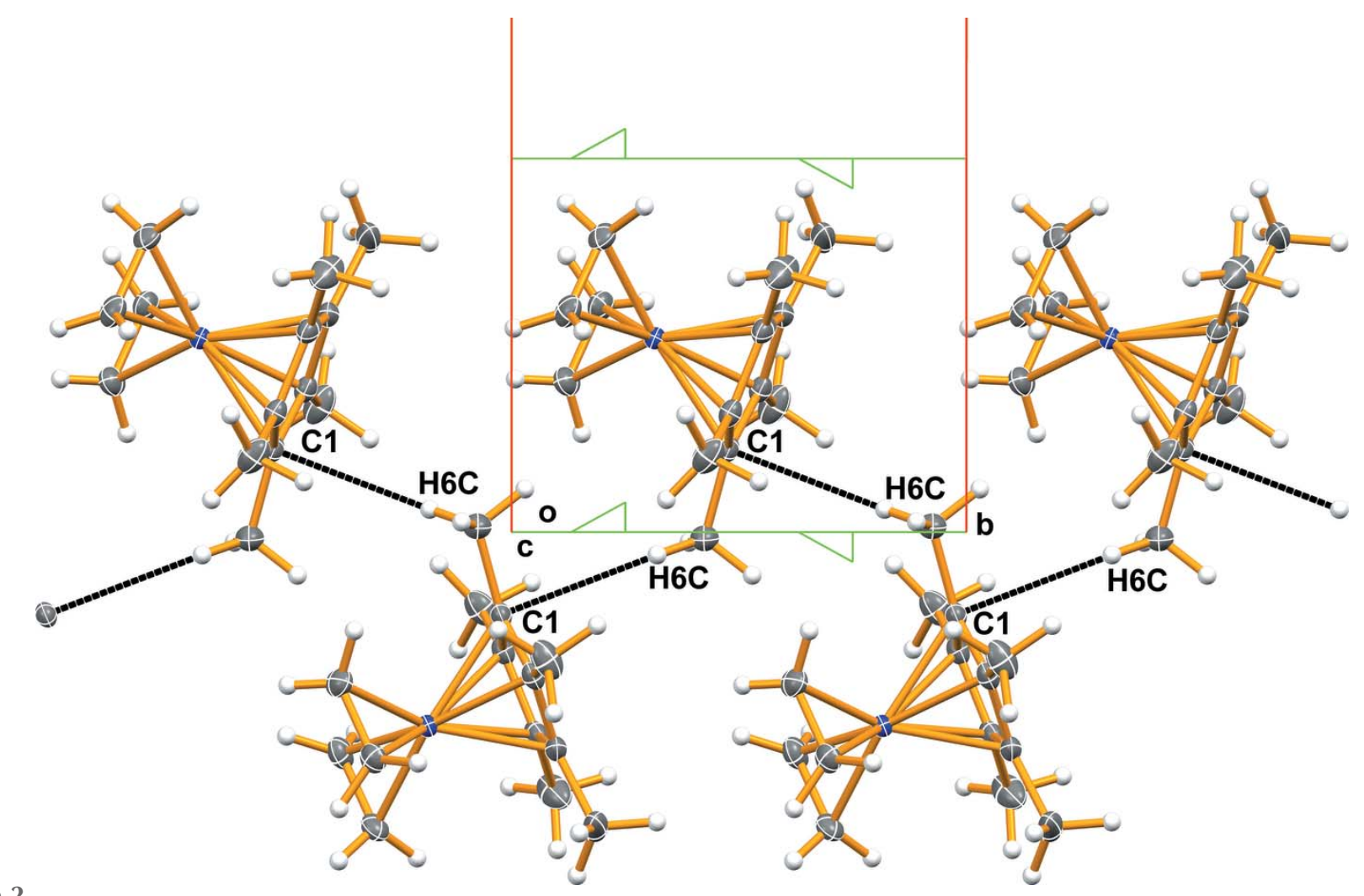

Figure 2

Packing diagram of the title compound, viewed down the $c$ axis, showing a chain formed by $\mathrm{C}-\mathrm{H} \cdots \pi$ interactions. Dotted lines show the shortest $\mathrm{C}-$ $\mathrm{H}$.. C contact involved in the interaction. Ellipsoids are drawn at the $50 \%$ probability level. The $2_{1}$ screw axes (green) are also shown. 
tallizes in the monoclinic space group $P 2_{1} / c$ with unit-cell dimensions of $a=12.5561$ (5), $b=7.3323$ (3), $c=14.7157$ (6) $\AA$ and $\beta=108.3520(10)^{\circ}$ at $100 \mathrm{~K}$.

\section{Synthesis and crystallization}

The title compound was prepared by reduction of $\left[\mathrm{Co}_{2}\left(\mathrm{C}_{10} \mathrm{H}_{15}\right)_{2}(\mu-\mathrm{Cl})_{2}\right]$ (Koelle et al., 1986) under ethylene. This procedure is an adaptation of that reported by Nicholls \& Spencer (1990). All solvents were degassed by purging with nitrogen and dried by passing through activated $\mathrm{Al}_{2} \mathrm{O}_{3}$. A $1 \%$ $\mathrm{Na}$ amalgam was prepared by addition of $\mathrm{Na}$ (305 mg, $13.3 \mathrm{mmol})$ in small portions to mercury $(30.5 \mathrm{~g})$ in a Schlenk flask equipped with a stir bar and rubber septum under a nitrogen atmosphere. The sodium was allowed to disperse completely between additions. Gentle heating with a heat gun may be required to initiate the process after the first addition. The $\mathrm{Na}$ amalgam was cooled to room temperature. THF $(100 \mathrm{ml})$ was added to the Schlenk flask, followed by gently bubbling ethylene through the system via a needle for $20 \mathrm{~min}$ to ensure saturation. Previously prepared $\left[\mathrm{Co}_{2}\left(\mathrm{C}_{10} \mathrm{H}_{15}\right)(\mu\right.$ $\mathrm{Cl})_{2}$ ] $(2.77 \mathrm{~g}, 6.0 \mathrm{mmol})$ was removed from the glovebox and rapidly added to the Schlenk flask under a nitrogen purge. The ethylene was bubbled through the THF for an additional $10 \mathrm{~min}$, then the needle was moved to a position $\mathrm{ca} 1 \mathrm{~cm}$ above the solution surface to prevent clogging. The reaction was stirred under ethylene for a total of $1.5 \mathrm{~h}$. Over this timespan, the colour evolved from dark brown to a red/orange colour. At this point, the septum was replaced with a glass stopper and the solvent removed completely under vacuum. The evacuated flask was transferred to the glovebox where the product was taken up in pentane and filtered through Celite, taking care to separate the mercury. The solution was concentrated under vacuum in a Schlenk tube and then sealed with a greased glass stopper. The tube was removed from the glovebox and placed in a $193 \mathrm{~K}$ freezer overnight. The next day, the tube was removed from the freezer and immediately
Table 1

Experimental details.

\begin{tabular}{|c|c|}
\hline \multicolumn{2}{|l|}{ Crystal data } \\
\hline Chemical formula & {$\left[\mathrm{Co}\left(\mathrm{C}_{10} \mathrm{H}_{15}\right)\left(\mathrm{C}_{2} \mathrm{H}_{4}\right)_{2}\right]$} \\
\hline$M_{\mathrm{r}}$ & 250.25 \\
\hline Crystal system, space group & Monoclinic, $P 2_{1} / c$ \\
\hline Temperature $(\mathrm{K})$ & 125 \\
\hline$a, b, c(\AA)$ & $12.526(2), 7.2647(13), 14.712(3)$ \\
\hline$\beta\left(^{\circ}\right)$ & $107.860(2)$ \\
\hline$V\left(\AA^{3}\right)$ & $1274.3(4)$ \\
\hline$Z$ & 4 \\
\hline Radiation type & Мо $K \alpha$ \\
\hline$\mu\left(\mathrm{mm}^{-1}\right)$ & 1.31 \\
\hline Crystal size $(\mathrm{mm})$ & $0.23 \times 0.12 \times 0.10$ \\
\hline \multicolumn{2}{|l|}{ Data collection } \\
\hline Diffractometer & Bruker APEXII CCD \\
\hline Absorption correction & $\begin{array}{l}\text { Multi-scan (SADABS; Bruker, } \\
\text { 2009) }\end{array}$ \\
\hline$T_{\min }, T_{\max }$ & $0.524,0.746$ \\
\hline $\begin{array}{l}\text { No. of measured, independent and } \\
\text { observed }[I>2 \sigma(I)] \text { reflections }\end{array}$ & $14405,3165,2684$ \\
\hline$R_{\text {int }}$ & 0.084 \\
\hline$(\sin \theta / \lambda)_{\max }\left(\AA^{-1}\right)$ & 0.676 \\
\hline \multicolumn{2}{|l|}{ Refinement } \\
\hline$R\left[F^{2}>2 \sigma\left(F^{2}\right)\right], w R\left(F^{2}\right), S$ & $0.039,0.106,1.06$ \\
\hline No. of reflections & 3165 \\
\hline No. of parameters & 165 \\
\hline $\mathrm{H}$-atom treatment & $\begin{array}{l}\mathrm{H} \text { atoms treated by a mixture of } \\
\text { independent and constrained } \\
\text { refinement }\end{array}$ \\
\hline$\Delta \rho_{\max }, \Delta \rho_{\min }\left(\mathrm{e} \AA^{-3}\right)$ & $0.41,-0.51$ \\
\hline
\end{tabular}

Computer programs: APEX2 and SAINT (Bruker, 2008), SHELXT (Sheldrick, 2015a), SHELXL2014 (Sheldrick, 2015b) and Mercury (Macrae et al., 2006).

immersed in a dry ice/acetone bath and placed under inert atmosphere on the Schlenk line. The solvent was removed by canula transfer at low temperature to isolate the title compound $(1.8 \mathrm{~g}, 60 \%)$ as dark-red rectangular crystals. The product was dried under vacuum and transferred to the glovebox where it was stored at $233 \mathrm{~K}$. The NMR spectroscopic data is identical to that previously reported (Beevor et al., 1981; Nicholls \& Spencer, 1990).
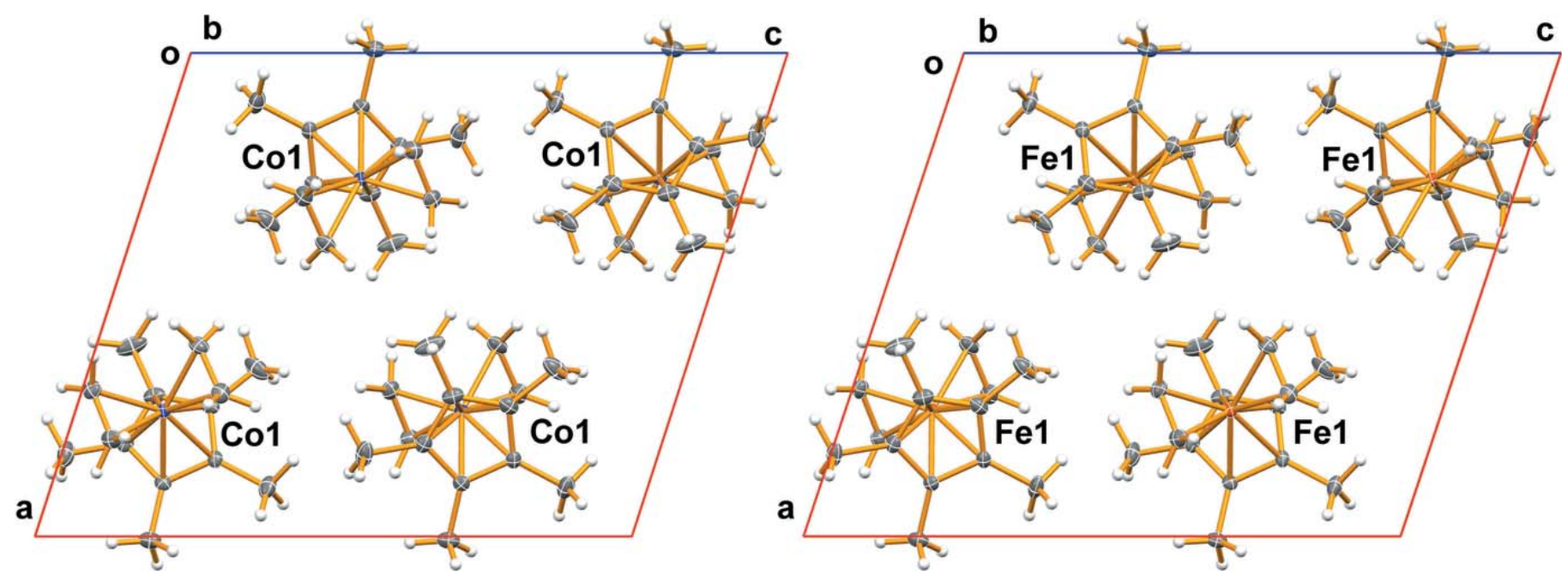

Figure 3

Comparison diagram of the isostructural $\mathrm{Cp} * \mathrm{Co}\left(\mathrm{CH}_{2} \mathrm{CH}_{2}\right)_{2}$ (left) and $\mathrm{Cp} * \mathrm{Fe}\left(\mathrm{CH}_{2} \mathrm{CH}_{2}\right)_{2}$ (right) unit cells, viewed down the $b$ axis. 


\section{Refinement}

Crystal data, data collection, and structure refinement details are summarized in Table 1. The $\mathrm{H}$ atoms of the methyl groups were included at geometrically idealized positions $(\mathrm{C}-\mathrm{H}=$ $0.98 \AA$ ) and were treated as riding, with $U_{\text {iso }}(\mathrm{H})=1.5 U_{\text {eq }}(\mathrm{C})$. The $\mathrm{H}$ atoms of the ethylene groups were located in a difference-Fourier map and their positions were freely refined, while their $U_{\text {iso }}(\mathrm{H})$ values were set to be equal to $1.2 U_{\text {eq }}$ of the parent carbon atom.

\section{Acknowledgements}

Financial support from the Canada Foundation for Innovation (CFI), the Faculties of Science and Graduate Studies and Research of Saint Mary's University and ACEnet (ACEnet Summer Research Fellowship to CDR) is gratefully acknowledged.

\section{References}

Beevor, R. G., Frith, S. A. \& Spencer, J. L. (1981). J. Organomet. Chem. 221, C25-C27.

Broere, D. L. J. \& Ruijter, E. (2012). Synthesis, 44, 2639-2672.

Brookhart, M., Grant, B. E., Lenges, C. P., Prosenc, M. H. \& White, P. S. (2000). Angew. Chem. Int. Ed. 39, 1676-1679.

Bruker (2008). APEX2 and SAINT. Bruker AXS Inc., Madison, Wisconsin, USA.

Bruker (2009). SADABS. Bruker AXS Inc., Madison, Wisconsin, USA.
Chan, N. H., Roache, J. H. \& Jones, W. D. (2015). Inorg. Chim. Acta, 437, 36-40.

Dosa, P. I., Whitener, G. D., Vollhardt, K. P. C., Bond, A. D. \& Teat, S. J. (2002). Org. Lett. 4, 2075-2078.

Fürstner, A., Martin, R., Krause, H., Seidel, G., Goddard, R. \& Lehmann, C. W. (2008). J. Am. Chem. Soc. 130, 8773-8787.

Groom, C. R., Bruno, I. J., Lightfoot, M. P. \& Ward, S. C. (2016). Acta Cryst. B72, 171-179.

Holmes, D., Lee, S. Y., Lotz, S. D., Nguyen, S., Schaller, G. R., Schmidt-Radde, R. H. \& Vollhardt, K. P. C. (2015). Synthesis, 47, 2038-2054.

Jones, W. D. \& Chin, R. M. (1994). J. Organomet. Chem. 472, 311-316.

Koelle, U., Fuss, B., Belting, M. \& Raabe, E. (1986). Organometallics, 5, 980-987.

Lenges, C. P., Brookhart, M. \& Grant, B. E. (1997). J. Organomet. Chem. 528, 199-203.

Lenges, C. P., White, P. S. \& Brookhart, M. (1998). J. Am. Chem. Soc. 120, 6965-6979.

Lenges, C. P., White, P. S., Marshall, W. J. \& Brookhart, M. (2000). Organometallics, 19, 1247-1254.

Macrae, C. F., Edgington, P. R., McCabe, P., Pidcock, E., Shields, G. P., Taylor, R., Towler, M. \& van de Streek, J. (2006). J. Appl. Cryst. 39, 453-457.

Nicholls, J. C. \& Spencer, J. L. (1990). Inorg. Synth. 28, 278-280.

Sheldrick, G. M. (2015a). Acta Cryst. A71, 3-8.

Sheldrick, G. M. (2015b). Acta Cryst. C71, 3-8.

Witherell, R. D., Ylijoki, K. E. O. \& Stryker, J. M. (2008). J. Am. Chem. Soc. 130, 2176-2177.

Ylijoki, K. E. O., Kirk, A. D., Böcklein, S., Witherell, R. D. \& Stryker, J. M. (2015). Organometallics, 34, 3335-3357.

Ylijoki, K. E. O., Witherell, R. D., Kirk, A. D., Böcklein, S., Lofstrand, V. A., McDonald, R., Ferguson, M. J. \& Stryker, J. M. (2009). Organometallics, 28, 6807-6822. 


\section{supporting information}

Acta Cryst. (2016). E72, 1301-1304 [https://doi.org/10.1107/S2056989016012822]

\section{Crystal structure of bis( $\eta^{2}$-ethylene $)\left(\eta^{5}\right.$-pentamethylcyclopentadienyl)cobalt}

\section{Chandika D. Ramful, Katherine N. Robertson and Kai E. O. Ylijoki}

Computing details

Data collection: APEX2 (Bruker, 2008); cell refinement: SAINT (Bruker, 2008); data reduction: SAINT (Bruker, 2008); program(s) used to solve structure: SHELXT (Sheldrick, 2015a); program(s) used to refine structure: SHELXL2014 (Sheldrick, 2015b); molecular graphics: Mercury (Macrae et al., 2006); software used to prepare material for publication: SHELXL2014 (Sheldrick, 2015b).

Bis $\left(\eta^{2}\right.$-ethylene $)\left(\eta^{5}\right.$-pentamethylcyclopentadienyl) cobalt

Crystal data

$\left[\mathrm{Co}\left(\mathrm{C}_{10} \mathrm{H}_{15}\right)\left(\mathrm{C}_{2} \mathrm{H}_{4}\right)_{2}\right]$

$M_{r}=250.25$

Monoclinic, $P 2{ }_{1} / c$

$a=12.526(2) \AA$

$b=7.2647$ (13) $\AA$

$c=14.712(3) \AA$

$\beta=107.860(2)^{\circ}$

$V=1274.3(4) \AA^{3}$

$Z=4$

Data collection

Bruker APEXII CCD diffractometer

Radiation source: sealed tube

Graphite monochromator

$\varphi$ and $\omega$ scans

Absorption correction: multi-scan

(SADABS; Bruker, 2009)

$T_{\min }=0.524, T_{\max }=0.746$

Refinement

Refinement on $F^{2}$

Least-squares matrix: full

$R\left[F^{2}>2 \sigma\left(F^{2}\right)\right]=0.039$

$w R\left(F^{2}\right)=0.106$

$S=1.06$

3165 reflections

165 parameters

0 restraints
$F(000)=536$

$D_{\mathrm{x}}=1.304 \mathrm{Mg} \mathrm{m}^{-3}$

Mo $K \alpha$ radiation, $\lambda=0.71073 \AA$

Cell parameters from 7968 reflections

$\theta=2.9-28.7^{\circ}$

$\mu=1.31 \mathrm{~mm}^{-1}$

$T=125 \mathrm{~K}$

Irregular, orange-brown

$0.23 \times 0.12 \times 0.10 \mathrm{~mm}$

14405 measured reflections

3165 independent reflections

2684 reflections with $I>2 \sigma(I)$

$R_{\text {int }}=0.084$

$\theta_{\text {max }}=28.7^{\circ}, \theta_{\min }=2.9^{\circ}$

$h=-16 \rightarrow 16$

$k=-9 \rightarrow 9$

$l=-19 \rightarrow 18$

Hydrogen site location: mixed

$\mathrm{H}$ atoms treated by a mixture of independent and constrained refinement

$w=1 /\left[\sigma^{2}\left(F_{\mathrm{o}}^{2}\right)+(0.0509 P)^{2}+0.0513 P\right]$

where $P=\left(F_{\mathrm{o}}{ }^{2}+2 F_{\mathrm{c}}{ }^{2}\right) / 3$

$(\Delta / \sigma)_{\max }=0.001$

$\Delta \rho_{\max }=0.41 \mathrm{e} \AA^{-3}$

$\Delta \rho_{\min }=-0.51$ e $\AA^{-3}$ 


\section{Special details}

Geometry. All esds (except the esd in the dihedral angle between two 1.s. planes) are estimated using the full covariance matrix. The cell esds are taken into account individually in the estimation of esds in distances, angles and torsion angles; correlations between esds in cell parameters are only used when they are defined by crystal symmetry. An approximate (isotropic) treatment of cell esds is used for estimating esds involving l.s. planes.

Fractional atomic coordinates and isotropic or equivalent isotropic displacement parameters $\left(\AA^{2}\right)$

\begin{tabular}{|c|c|c|c|c|}
\hline & $x$ & $y$ & $z$ & $U_{\text {iso }} * / U_{\text {eq }}$ \\
\hline Co1 & $0.25940(2)$ & $0.31555(3)$ & $0.35283(2)$ & $0.01378(11)$ \\
\hline $\mathrm{C} 1$ & $0.11064(16)$ & $0.4768(2)$ & $0.31455(13)$ & $0.0162(4)$ \\
\hline $\mathrm{C} 2$ & $0.15937(16)$ & $0.4812(2)$ & $0.23996(13)$ & $0.0172(4)$ \\
\hline $\mathrm{C} 3$ & 0.27067 (17) & $0.5547(3)$ & $0.27741(15)$ & $0.0206(4)$ \\
\hline $\mathrm{C} 4$ & $0.29067(17)$ & $0.5974(3)$ & $0.37588(15)$ & $0.0204(4)$ \\
\hline $\mathrm{C} 5$ & $0.19206(17)$ & $0.5474(3)$ & $0.39890(14)$ & $0.0184(4)$ \\
\hline C6 & $-0.00806(17)$ & $0.4243(3)$ & $0.30531(16)$ & $0.0247(4)$ \\
\hline H6A & -0.0577 & 0.5290 & 0.2802 & $0.037^{*}$ \\
\hline H6B & -0.0135 & 0.3899 & 0.3681 & $0.037^{*}$ \\
\hline $\mathrm{H} 6 \mathrm{C}$ & -0.0305 & 0.3197 & 0.2615 & $0.037^{*}$ \\
\hline $\mathrm{C} 7$ & $0.10181(19)$ & $0.4326(3)$ & $0.13701(15)$ & $0.0276(5)$ \\
\hline H7A & 0.0436 & 0.3403 & 0.1334 & $0.041^{*}$ \\
\hline H7B & 0.1569 & 0.3826 & 0.1086 & $0.041^{*}$ \\
\hline $\mathrm{H} 7 \mathrm{C}$ & 0.0675 & 0.5433 & 0.1020 & $0.041^{*}$ \\
\hline $\mathrm{C} 8$ & $0.3474(2)$ & $0.5936(3)$ & $0.2190(2)$ & $0.0355(6)$ \\
\hline $\mathrm{H} 8 \mathrm{~A}$ & 0.3266 & 0.7112 & 0.1856 & $0.053^{*}$ \\
\hline $\mathrm{H} 8 \mathrm{~B}$ & 0.3408 & 0.4949 & 0.1722 & $0.053^{*}$ \\
\hline $\mathrm{H} 8 \mathrm{C}$ & 0.4250 & 0.6003 & 0.2610 & $0.053^{*}$ \\
\hline C9 & $0.3941(2)$ & $0.6847(3)$ & $0.4412(2)$ & $0.0350(6)$ \\
\hline H9A & 0.4597 & 0.6437 & 0.4237 & $0.052^{*}$ \\
\hline H9B & 0.4027 & 0.6487 & 0.5073 & $0.052^{*}$ \\
\hline $\mathrm{H} 9 \mathrm{C}$ & 0.3877 & 0.8189 & 0.4354 & $0.052^{*}$ \\
\hline $\mathrm{C} 10$ & $0.1708(2)$ & $0.5774(3)$ & $0.49277(15)$ & $0.0303(5)$ \\
\hline H10A & 0.1248 & 0.6880 & 0.4889 & $0.045^{*}$ \\
\hline H10B & 0.2424 & 0.5931 & 0.5432 & $0.045^{*}$ \\
\hline $\mathrm{H} 10 \mathrm{C}$ & 0.1311 & 0.4707 & 0.5075 & $0.045^{*}$ \\
\hline C11 & $0.39512(19)$ & $0.1994(3)$ & $0.32878(17)$ & $0.0231(5)$ \\
\hline H11A & $0.436(2)$ & $0.292(3)$ & $0.3065(18)$ & $0.028^{*}$ \\
\hline H11B & $0.440(2)$ & $0.120(3)$ & $0.3815(18)$ & $0.028^{*}$ \\
\hline $\mathrm{C} 12$ & 0.29566 (19) & $0.1242(3)$ & $0.26683(16)$ & $0.0241(4)$ \\
\hline H12A & $0.269(2)$ & $0.156(3)$ & $0.201(2)$ & $0.029 *$ \\
\hline H12B & $0.273(2)$ & $0.005(4)$ & $0.2803(18)$ & $0.029^{*}$ \\
\hline $\mathrm{C} 13$ & $0.30282(19)$ & $0.1963(3)$ & $0.48330(16)$ & $0.0214(4)$ \\
\hline H13A & $0.370(2)$ & $0.121(3)$ & $0.4991(17)$ & $0.026^{*}$ \\
\hline H13B & $0.309(2)$ & $0.294(3)$ & $0.5351(19)$ & $0.026^{*}$ \\
\hline $\mathrm{C} 14$ & $0.20163(19)$ & $0.1212(3)$ & $0.42396(16)$ & $0.0234(4)$ \\
\hline H14A & $0.132(2)$ & $0.154(3)$ & $0.4317(19)$ & $0.028^{*}$ \\
\hline H14B & $0.205(2)$ & $0.008(4)$ & $0.4038(18)$ & $0.028^{*}$ \\
\hline
\end{tabular}


Atomic displacement parameters $\left(\AA^{2}\right)$

\begin{tabular}{lllllll}
\hline & $U^{11}$ & $U^{22}$ & $U^{\beta 3}$ & $U^{12}$ & $U^{13}$ & $U^{23}$ \\
\hline Co1 & $0.01325(17)$ & $0.01235(16)$ & $0.01530(17)$ & $0.00046(8)$ & $0.00374(11)$ & $0.00026(9)$ \\
C1 & $0.0174(9)$ & $0.0125(8)$ & $0.0188(9)$ & $0.0025(7)$ & $0.0059(8)$ & $0.0006(7)$ \\
C2 & $0.0205(9)$ & $0.0151(9)$ & $0.0163(9)$ & $0.0042(7)$ & $0.0060(7)$ & $0.0018(7)$ \\
C3 & $0.0200(10)$ & $0.0164(9)$ & $0.0272(10)$ & $0.0046(7)$ & $0.0100(8)$ & $0.0071(8)$ \\
C4 & $0.0178(9)$ & $0.0124(9)$ & $0.0275(10)$ & $0.0001(7)$ & $0.0021(8)$ & $0.0011(7)$ \\
C5 & $0.0215(10)$ & $0.0147(9)$ & $0.0176(9)$ & $0.0042(7)$ & $0.0041(8)$ & $-0.0002(7)$ \\
C6 & $0.0180(10)$ & $0.0222(10)$ & $0.0337(12)$ & $0.0002(8)$ & $0.0075(9)$ & $0.0006(9)$ \\
C7 & $0.0335(12)$ & $0.0297(11)$ & $0.0173(10)$ & $0.0106(9)$ & $0.0043(9)$ & $0.0017(8)$ \\
C8 & $0.0339(13)$ & $0.0332(13)$ & $0.0490(15)$ & $0.0080(10)$ & $0.0268(12)$ & $0.0185(11)$ \\
C9 & $0.0254(12)$ & $0.0168(11)$ & $0.0490(16)$ & $-0.0011(8)$ & $-0.0088(11)$ & $-0.0036(9)$ \\
C10 & $0.0424(14)$ & $0.0288(11)$ & $0.0194(10)$ & $0.0120(10)$ & $0.0092(10)$ & $-0.0029(9)$ \\
C11 & $0.0208(11)$ & $0.0213(10)$ & $0.0304(12)$ & $0.0051(8)$ & $0.0128(9)$ & $0.0003(8)$ \\
C12 & $0.0282(11)$ & $0.0195(10)$ & $0.0242(11)$ & $0.0059(9)$ & $0.0073(9)$ & $-0.0038(8)$ \\
C13 & $0.0237(11)$ & $0.0191(10)$ & $0.0201(10)$ & $0.0024(8)$ & $0.0047(9)$ & $0.0053(7)$ \\
C14 & $0.0223(11)$ & $0.0200(10)$ & $0.0279(11)$ & $-0.0027(8)$ & $0.0078(9)$ & $0.0060(9)$ \\
& & & & & & \\
\hline
\end{tabular}

Geometric parameters $\left(\AA,{ }^{\circ}\right)$

\begin{tabular}{|c|c|c|c|}
\hline $\mathrm{Co} 1-\mathrm{C} 14$ & $2.019(2)$ & $\mathrm{C} 7-\mathrm{H} 7 \mathrm{~A}$ & 0.9800 \\
\hline $\mathrm{Co} 1-\mathrm{C} 13$ & $2.022(2)$ & $\mathrm{C} 7-\mathrm{H} 7 \mathrm{~B}$ & 0.9800 \\
\hline $\mathrm{Co} 1-\mathrm{C} 12$ & $2.023(2)$ & $\mathrm{C} 7-\mathrm{H} 7 \mathrm{C}$ & 0.9800 \\
\hline $\mathrm{Co} 1-\mathrm{C} 11$ & $2.024(2)$ & $\mathrm{C} 8-\mathrm{H} 8 \mathrm{~A}$ & 0.9800 \\
\hline $\mathrm{Co} 1-\mathrm{C} 5$ & 2.0875 (19) & $\mathrm{C} 8-\mathrm{H} 8 \mathrm{~B}$ & 0.9800 \\
\hline $\mathrm{Co} 1-\mathrm{C} 3$ & 2.0894 (19) & $\mathrm{C} 8-\mathrm{H} 8 \mathrm{C}$ & 0.9800 \\
\hline $\mathrm{Co} 1-\mathrm{C} 4$ & $2.093(2)$ & $\mathrm{C} 9-\mathrm{H} 9 \mathrm{~A}$ & 0.9800 \\
\hline $\mathrm{Co} 1-\mathrm{C} 2$ & $2.1214(19)$ & $\mathrm{C} 9-\mathrm{H} 9 \mathrm{~B}$ & 0.9800 \\
\hline $\mathrm{Co} 1-\mathrm{C} 1$ & $2.1256(18)$ & $\mathrm{C} 9-\mathrm{H} 9 \mathrm{C}$ & 0.9800 \\
\hline $\mathrm{C} 1-\mathrm{C} 2$ & $1.410(3)$ & $\mathrm{C} 10-\mathrm{H} 10 \mathrm{~A}$ & 0.9800 \\
\hline $\mathrm{C} 1-\mathrm{C} 5$ & $1.438(3)$ & $\mathrm{C} 10-\mathrm{H} 10 \mathrm{~B}$ & 0.9800 \\
\hline $\mathrm{C} 1-\mathrm{C} 6$ & $1.500(3)$ & $\mathrm{C} 10-\mathrm{H} 10 \mathrm{C}$ & 0.9800 \\
\hline $\mathrm{C} 2-\mathrm{C} 3$ & $1.436(3)$ & $\mathrm{C} 11-\mathrm{C} 12$ & $1.409(3)$ \\
\hline $\mathrm{C} 2-\mathrm{C} 7$ & $1.506(3)$ & C11-H11A & $0.97(3)$ \\
\hline $\mathrm{C} 3-\mathrm{C} 4$ & $1.427(3)$ & C11-H11B & $0.99(3)$ \\
\hline $\mathrm{C} 3-\mathrm{C} 8$ & $1.500(3)$ & $\mathrm{C} 12-\mathrm{H} 12 \mathrm{~A}$ & $0.95(3)$ \\
\hline $\mathrm{C} 4-\mathrm{C} 5$ & $1.425(3)$ & $\mathrm{C} 12-\mathrm{H} 12 \mathrm{~B}$ & $0.95(3)$ \\
\hline $\mathrm{C} 4-\mathrm{C} 9$ & $1.497(3)$ & $\mathrm{C} 13-\mathrm{C} 14$ & $1.410(3)$ \\
\hline $\mathrm{C} 5-\mathrm{C} 10$ & $1.501(3)$ & $\mathrm{C} 13-\mathrm{H} 13 \mathrm{~A}$ & $0.98(3)$ \\
\hline C6-H6A & 0.9800 & C13-H13B & $1.03(2)$ \\
\hline C6-H6B & 0.9800 & $\mathrm{C} 14-\mathrm{H} 14 \mathrm{~A}$ & $0.95(3)$ \\
\hline $\mathrm{C} 6-\mathrm{H} 6 \mathrm{C}$ & 0.9800 & C14-H14B & $0.88(3)$ \\
\hline $\mathrm{C} 14-\mathrm{Co} 1-\mathrm{C} 13$ & $40.83(9)$ & $\mathrm{C} 4-\mathrm{C} 5-\mathrm{C} 1$ & $108.86(17)$ \\
\hline $\mathrm{C} 14-\mathrm{Co} 1-\mathrm{C} 12$ & $91.64(10)$ & $\mathrm{C} 4-\mathrm{C} 5-\mathrm{C} 10$ & $126.40(19)$ \\
\hline $\mathrm{C} 13-\mathrm{Co} 1-\mathrm{C} 12$ & $104.64(9)$ & $\mathrm{C} 1-\mathrm{C} 5-\mathrm{C} 10$ & $124.54(19)$ \\
\hline $\mathrm{C} 14-\mathrm{Co} 1-\mathrm{C} 11$ & $104.65(9)$ & $\mathrm{C} 4-\mathrm{C} 5-\mathrm{Co} 1$ & $70.28(11)$ \\
\hline
\end{tabular}




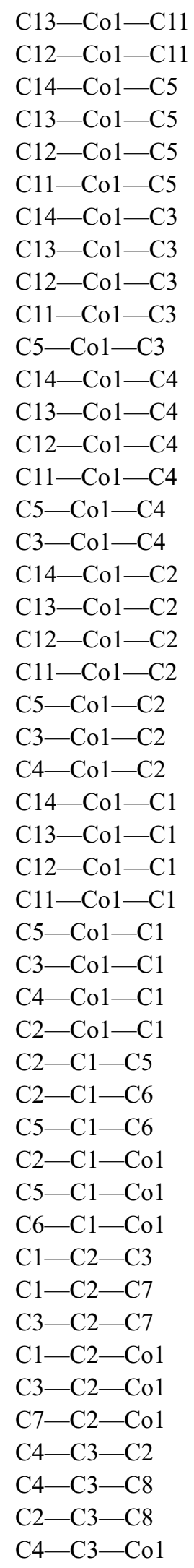

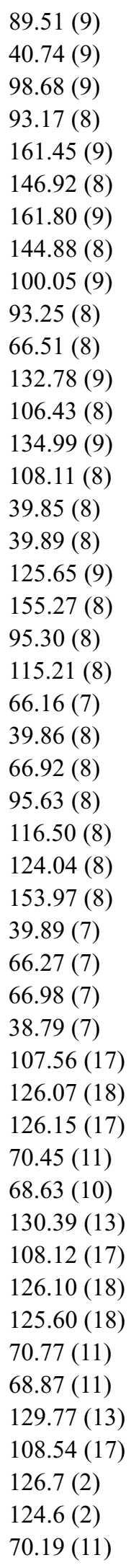

$\begin{array}{ll}\text { C1-C5-Co1 } & 71.48(10) \\ \text { C10-C5-Co1 } & 128.29(14) \\ \text { C1-C6-H6A } & 109.5 \\ \text { C1-C6-H6B } & 109.5 \\ \text { H6A-C6-H6B } & 109.5 \\ \text { C1-C6-H6C } & 109.5 \\ \text { H6A-C6-H6C } & 109.5 \\ \text { H6B-C6-H6C } & 109.5 \\ \text { C2-C7-H7A } & 109.5 \\ \text { C2-C7-H7B } & 109.5 \\ \text { H7A-C7-H7B } & 109.5 \\ \text { C2-C7-H7C } & 109.5 \\ \text { H7A-C7-H7C } & 109.5 \\ \text { H7B-C7-H7C } & 109.5 \\ \text { C3-C8-H8A } & 109.5 \\ \text { C3-C8-H8B } & 109.5 \\ \text { H8A-C8-H8B } & 109.5 \\ \text { C3-C8-H8C } & 109.5 \\ \text { H8A-C8-H8C } & 109.5 \\ \text { H8B-C8-H8C } & 109.5 \\ \text { C4-C9-H9A } & 109.5 \\ \text { C4-C9-H9B } & 109.5 \\ \text { H9A-C9-H9B } & 109.5 \\ \text { C4-C9-H9C } & 109.5 \\ \text { H9A-C9-H9C } & 109.5 \\ \text { H9B-C9-H9C } & 109.5 \\ \text { C5-C10-H10A } & 109.5 \\ \text { C5-C10-H10B } & 109.5 \\ \text { H10A-C10-H10B } & 109.5 \\ \text { C5-C10-H10C } & 109.5 \\ \text { H10A-C10-H10C } & 109.5 \\ \text { H10B-C10-H10C } & 109.5 \\ \text { C12-C11-Co1 } & 69.58(12) \\ \text { C12-C11-H11A } & 120.6(16) \\ \text { Co1-C11-H11A } & 109.4(15) \\ \text { C12-C11-H11B } & 117.1(15) \\ \text { Co1-C11-H11B } & 114.1(14) \\ \text { H11A-C11-H11B } & 116(2) \\ \text { C11-C12-Co1 } & 69.67(12) \\ \text { C11-C12-H12A } & 122.1(16) \\ \text { Co1-C12-H12A } & 113.1(15) \\ \text { C11-C12-H12B } & 118.7(15) \\ \text { Co1-C12-H12B } & 110.9(15) \\ \text { H12A-C12-H12B } & 113(2) \\ \text { C14-C13-Co1 } & \\ \text { C14-C13-H13A } & \text { Co1-C13-H13A } \\ \text { C14-C13-H13B } & \\ & \end{array}$


$71.27(11)$

$128.37(15)$

$106.90(17)$

$126.8(2)$

$126.3(2)$

$69.87(11)$

$69.92(11)$

$126.91(14)$

$-0.1(2)$

$174.76(17)$

$-58.94(13)$

$-175.46(17)$

-0.5 (3)

$125.75(19)$

$58.80(12)$

$-126.29(18)$

-0.4 (2)

$174.89(18)$

$-60.58(13)$

$-175.64(18)$

$-0.3(3)$

$124.2(2)$

$60.13(13)$

-124.53 (19)

$0.9(2)$

$175.94(19)$

$-60.39(13)$

$-177.08(18)$

$$
\begin{aligned}
& \text { Co1-C13-H13B } \\
& \text { H13A-C13-H13B } \\
& \text { C13-C14-Co1 } \\
& \text { C13-C14-H14A } \\
& \text { Co1-C14-H14A } \\
& \text { C13-C14-H14B } \\
& \text { Co1-C14-H14B } \\
& \text { H14A-C14-H14B }
\end{aligned}
$$

$\mathrm{C} 8-\mathrm{C} 3-\mathrm{C} 4-\mathrm{C} 9$

$\mathrm{Co} 1-\mathrm{C} 3-\mathrm{C} 4-\mathrm{C} 9$

$\mathrm{C} 2-\mathrm{C} 3-\mathrm{C} 4-\mathrm{Co} 1$

$\mathrm{C} 8-\mathrm{C} 3-\mathrm{C} 4-\mathrm{Co} 1$

$\mathrm{C} 3-\mathrm{C} 4-\mathrm{C} 5-\mathrm{C} 1$

$\mathrm{C} 9-\mathrm{C} 4-\mathrm{C} 5-\mathrm{C} 1$

$\mathrm{Co} 1-\mathrm{C} 4-\mathrm{C} 5-\mathrm{C} 1$

$\mathrm{C} 3-\mathrm{C} 4-\mathrm{C} 5-\mathrm{C} 10$

$\mathrm{C} 9-\mathrm{C} 4-\mathrm{C} 5-\mathrm{C} 10$

$\mathrm{Co} 1-\mathrm{C} 4-\mathrm{C} 5-\mathrm{C} 10$

$\mathrm{C} 3-\mathrm{C} 4-\mathrm{C} 5-\mathrm{Co} 1$

$\mathrm{C} 9-\mathrm{C} 4-\mathrm{C} 5-\mathrm{Co} 1$

$\mathrm{C} 2-\mathrm{C} 1-\mathrm{C} 5-\mathrm{C} 4$

$\mathrm{C} 6-\mathrm{C} 1-\mathrm{C} 5-\mathrm{C} 4$

$\mathrm{Co} 1-\mathrm{C} 1-\mathrm{C} 5-\mathrm{C} 4$

$\mathrm{C} 2-\mathrm{C} 1-\mathrm{C} 5-\mathrm{C} 10$

$\mathrm{C} 6-\mathrm{C} 1-\mathrm{C} 5-\mathrm{C} 10$

$\mathrm{Co} 1-\mathrm{C} 1-\mathrm{C} 5-\mathrm{C} 10$

$\mathrm{C} 2-\mathrm{C} 1-\mathrm{C} 5-\mathrm{Co} 1$

$\mathrm{C} 6-\mathrm{C} 1-\mathrm{C} 5-\mathrm{Co} 1$
$110.3(13)$

111 (2)

$69.72(12)$

$121.0(16)$

$112.1(15)$

116.1 (16)

114.5 (17)

$115(2)$

$-2.0(3)$

121.7 (2)

$61.26(13)$

$-123.7(2)$

$-1.0(2)$

$176.98(18)$

$-61.39(13)$

$-175.97(18)$

$2.0(3)$

$123.6(2)$

$60.42(13)$

$-121.6(2)$

0.7 (2)

$-174.21(18)$

60.64 (13)

$175.81(18)$

0.9 (3)

$-124.24(19)$

$-59.94(13)$

$125.15(18)$ 